Grundlagen der Kommunikation und Kognition Foundations of Communication and Cognition Herausgeber/Editors Roland Posner, Georg Meggle 



\section{Signs of Work}

Semiosis and Information Processing in Otganisations

Edited by

Berit Holmqvist

Peter Bøgh Andersen

Heinz Klein

Roland Posner

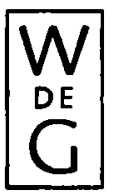

Walter de Gruyter - Berlin · New York 1996 
$\otimes$ Printed on acid-free paper which falls within the guidelines of the ANSI to ensure permanence and durability.

I.ibrary of Congress Cataloging-in-Publication Data

Signs of work : semiosis and information processing in organisations / edited by Berit Holmqvist ... [et al.].

p. $\quad \mathrm{cm}$. - (Grundlagen der Kommunikation und Kognition = Foundations of communication and cognition)

Includes bibliographical references and index.

ISBN 3-11-014095-0 (alk. paper)

1. Semiotics. 2. Communication in organizations. 3. Information storage and retrieval systems. I. Holmqvist, Berit. II. Series: Foundations of communication and cognition. P99.S4948 1996

$302.2-\mathrm{dc} 20$

Die Deutsche Bibliothek - Cataloging-in-Publication Data

Signs of work : semiosis and information processing in organisations / ed. by Berit Holmqvist ... - Berlin ; New York : de Gruyter, 1996

(Grundlagen der Kommunikation und Kognition)

ISBN 3-11-014095-0

NE: Holmqvist, Berit [Hrsg.]

(C) Copyright 1996 by Walter de Gruyter \& Co., D-10785 Berlin

All rights reserved, including those of translation into foreign languages. No part of this book may be reproduced or transmitted in any form or by any means, electronic or mechanical, including photocopy, recording or any information storage and retrieval system, without permission in writing from the publisher.

Printed in Germany

Printing: Werner Hildebrand, Berlin

Binding: Lüderity \& Bauer, Berlin

Cover Design: Rudolf Hübler, Berlin. 


\title{
Preface
}

\section{The Potential Contribution of Semiotics and Systems Theory to the Continuing Evolution of Information Systems Research}

\author{
HEINZ K. KLEIN
}

During the last decade information system research has grown dramatically to embrace the insights of several philosophical traditions. Both the scope and sophistication of information systems research have benefited from this. The purpose of this general introduction is to sketch how the papers collected in this unique monograph fit into it the intellectual landscape of research on information systems as we now know it from the Information Systems journals and how they might influence its future shape.

I will first propose some basic distinctions to characterize the major lines of thought which have sparked the debate in Information Systems. I will then use some of the papers as examples to illustrate the significance of these distinctions and to show how the semiotic viewpoint shared by this collection of papers can shed new light on certain aspects of the debate. Of course, any attempt to summarize a decade of literature debate in a paragraph or two runs the risk of using too broad brush blurring the details. However, I belief that this risk is worth taking if the simplifications throw a few basic points into relief that matter not only for the insider of information systems research, but also for those readers who approach this collection of papers from the vantage point of another discipline.

\section{Major Schools of Thought in Information Systems Research}

In this section I attempt to characterize some major lines of research in information systems in terms of their ontological and epistemological presuppositions. I am bringing up the subject of ontology and epistemology because it has sparked major debates in information systems research for more than a decade. It will also be useful to reflect where the papers in this volume stand on matters epistemological and ontological. 
Much of the fuel for the debate in information systems research has come from two fronts. One has emerged from rediscovering the significance of an old epistemological conflict about the nature of human knowledge in the context of information systems. This is natural as 'information' and knowledge are close cousins. Hence theories about knowledge and knowledge acquisition have direct implications for information systems design and use. The other front has formed around the question whether information systems design should help to stabilize the existing social order or help to discover and overcome its limitations for achieving a better way of life for all. The second conflict is often formulated with focus on organizational goals: Should information systems help to meet organizational goals as they are or help to reformulate these goals so that people can emancipate themselves from unwarranted constraints and better realize their potentials (Lyytinen 1992; Hirschheim, Klein 1994). More recently, a third front has been opened around the question whether information systems development can be anchored to given organizational structures which provide a persistent backdrop for systems development or whether structures are just fleeting images in a sea of change. In the former case, information systems can be analyzed as stabilizers in the latter case as vehicles of change which help the organization to maintain a sense of self when 'everything is in flux' (cf. Heracleitus' image that you can never swim in the same river twice).

The first front is essentially an epistemological and ontological one, the second an ethical one and the third one of research strategy. The first front is concerned with questions about the nature of reality and how we can learn about it. Researchers taking the position that the ultimate source of human knowledge is not experience but the constructions of the mind, are now often referred to as interpretivists or social constructivists. Those holding the opposing viewpoint, still the majority, are often labeled realists or positivists. Realists focus on the objective side of phenomena either on modeling things 'as they are' or as they are perceived by some subject. Ultimately they believe that there is a reality core which exists independently of any perceiving subject. Constructivist are interested in learning how the design and use of information systems intervenes in the construction of a socially shared reality. Whether or not a reality exists is moot because we can learn about it only through sharing our ideas of it, there is no immediate, privileged access to reality. Reducing the debate to its simplest denominator, one can say that the two sides differ in their beliefs on whether the ultimate source of knowledge is the human mind or the external world. One side believes that experience is the ultimate arbiter of truth, the other that "experience only confirms what the concept teaches" (Hegel).

The third front is directly influenced by the linguistic turn, i.e. namely that whatever counts as reality, to us humans is only accessible through the medium of language. Insofar as information systems affect language, they are 
reality constructors because they intervene in the process by which their users perceive, create and describe a shared reality.

As a discipline, Information Systems started in the sixties with an overwhelming bias towards realism and positivist research methodologies. Since the seventies it has made immense strides to put into place the intellectual foundations for a social constructivist approach to studying information systems by adopting principles and methods which are inspired by the philosophical schools of hermeneutics and phenomenology. Two good references documenting this broadening of the nature of information systems research are Nissen et al. (1990) and Hirschheim, Klein, Lyytinen (1995). In comparison to realism and constructivism in information systems research, the emergence theory of information systems is still in the making, in fact, there is not even a common terminology. It is closely connected to the proliferation of alternative or hybrid forms of organization in industry (cf. Applegate 1994). Besides emergent organizations (cf. Truex, Klein 1991; Baskerville, Travis, Truex 1992), such terms as virtual, self-designing or self-organizing, adaptive, autopoietic, and others are used to refer to constructs and phenomena which are at the core of this line of research (a cross-section of the pertinent literature is reviewed in Truex 1993:36).

\section{Two Conjectures}

The current volume takes a position in this debate which should be of interest to their participants regardless of their loyalty to one or the other philosophical school. For realists, any depiction or model of reality can only be as good as the language used to describe it and constructivists will agree that it is only through language that the mind can express its constructions. Moreover, since Tarski's analysis of semantical paradoxes, realists have accepted that natural language is the ultimate meta-language which is needed to give meaning and significance to any artificial language formalism, no matter how cleverly devised. Hence the study of how languages, be they formal or natural, 'empirically' affect signification in organizations, is of great interest to the realist camp in Information Systems. Constructivists on the other hand are predisposed to studying language as the medium which affects the social processes of sense-making and reality construction. For them, language is not only a medium of expression but an active force shaping the way the mind functions. From this vantage point, the following collection of papers also contains much food for thought papers to sharpen ideas on how all this happens and what the consequences are of the different ways in which it happens.

One critical shortcoming of the realist-constructivist debate has been the lack of communication between the two camps. The focus on language which 
this book suggests, could overcome the lack of a critical mass of ideas and concepts which is necessary to make any real communication possible among adherents to alternative philosophical traditions in research. The conceptual base which emerges from the following papers could be a milestone in overcoming this communication gap. From this perspective, this volume could also become a milestone on the further development of emergence theory because from its start, emergence theory has not only recognized the importance of language, but also drawn important inspirations from language theories in form of analogies and metaphors. In particular it has drawn on the theory of emergent grammar to gain insight how organizations could realize autopoiesis, i.e. how they are continually re-making themselves. ${ }^{1}$ As (with one exception) the following papers combine both conceptual and empirical work, they add detail and sophistication to understanding organizations as autopoietic systems in a form which should be of interest to realists and constructivists alike even though each may wish to take exceptions to some of the assumptions made.

The reference to autopoiesis points to a second shortcoming in the current debate in Information Systems, namely the way in which systems theory has been absorbed. With some notable research contributions (e.g. Winograd and Flores 1987), system theoretic notions in the information systems literature abound mostly in the form of concepts relating to system structure and boundary. Hence classical, physical systems theoretic notions are the most influential in the information systems literature. Meanwhile the theoretical discussion in systems theory has progressed from equilibrium seeking (structure maintaining) to autopoietic (self-making) and self-referential systems. Selfreferential systems maintain symbolic processes which determine ego and alter, i.e. which identify on a case by case basis the difference between inside and outside, us vs. them etc. To do this, self-referential systems must maintain and make use of identity, I.e. a symbolic image of self which they need to define what they are and what they are not. This transition from structure maintenance to identity preserving systems notions has amounted to no less than a paradigm shift in systems theory. Clearly with the penetration of computer-based information systems into all organizational processes, information systems

1 The meaning of "emergent grammar" is best explained by comparing it with the conventional notion of structural grammar. Structural grammar is conventionally understood as a set of rules which are stable across a multitude of conversations (diachronical) and act as shared language forms (templates) which make communication among speakers and hearers possible. Typically, structural grammar is construed to consist of a given set of rules which operate on fixed categories like nouns and verbs, specify how they can be modified through case, tense, modality, etc. and constrain the order in which they may appear to make well-formed sentences. Meanings are expressed by obeying these rules when speaking. Hopper (1988) has challenged this notion of (structural) grammar by documenting how grammars evolve with emergent cultures. Like cultures, they are temporal, emergent and disputed, always under negotiation and contingent on context of use, I.e. synchronical. For example, agreements about the meanings of words cannot be separated from agreements about the substantives issues under consideration. Likewise all language forms are as fluid as forms of life (Wittgenstein). 
must have become part of the identity formation processes determining what organizations are, the essence of their personality so to speak. Unfortunately, not much has been published on the consequences of this phenomenon for our understanding of the role information systems in real world contexts. Mapping the terrain of design and use of information systems from the perspective of modern system theory awaits discovery. It is therefore gratifying to note that the following collection of papers includes only both introductions to some of the newer conceptual developments of systems theory (e.g. Andersen, Jørgensen), as well as contributions which describe experiential implications of the paradigm shift in systems theory.

\section{Some Illustrations}

Each paper in this volume occupies a unique position in the matrix of the major intellectual forces which I have tried to characterize. Together they document a stage in the debate which has both methodological and substantive (contents) implications.

Methodologically, all papers merge elements from different philosophical positions into a unique blend which suits their varying purposes (this is explicitly recognized in Stamper's contribution). It would therefore not only be futile, but contrary to the spirit of this documentation of a certain stage in the discussion to ask which papers are more positivist and which are more contructivist. A more useful question is to ask what does the methodological stance which the author assumes and which may offend philosophical purists contribute to answering the research question to which the paper is addressed and how does it limit the results. For example, the apriori categories used in Klein and Truex impose a structure on the data which is contrary to the spirit of interpretivist research (in fact it is in part suggested by the reincarnation of structuralist grammatical analysis as proposed by Searle), but at the same time it points to possible generalizations which usually are beyond the reach of interpretivist field studies. Positivists may be concerned with the lack of statistical significance, but must recognize that hundreds of multivariate analysis have failed to shed light on the issues to which Klein and Truex speak. Similar questions can be raised about the other papers which mix formalism with field data, e.g. Madsen or Schmidt or Hørlück.

By the same token, none of the papers can be classified as 'critical', i.e. as directly confronting the issue of how language relates to human interests, how language use maintains power relations, distorts communication or related topics, yet most have 'windows' which shed critical light on questions of organizational participation (democracy) and politics. For example, Bærentsen's observation that machinists spend much time telling stories can be taken to 
mean that the main force keeping the power plant working is self-organization, that story telling is not just a past time lowering productivity but essential for maintaining identity and coordinated problem solving capacity. Clarke discusses the role of information systems as stabilizers of (obsolete) power relations and in Hørlück EDI is described as a tool which powerful buyers use to control smaller suppliers. This in turn may eventually change the structure of the market itself, presumably bringing it closer to the socialist ideal of a planned economy. Anderson's paper confronts the reader with a startling critical implication: if organizations are the result of self-organizing processes during which chaotic phases are inevitable, then the role of management tends to shrink from 'rational controllers and planners' to make-believe magicians who try to convince themselves and others that processes actually outside management control are still caused by rational management decisions. All of the above point to the conclusion that 'designing for democracy' is a rather complicated affair, especially if seen in the light of autopoiesis and selfreference.

In turning from questions of methodology to questions of substantive contents, it is interesting to note the vision which almost all papers share of the nature and role of information systems. With the exception of $B \varnothing \mathrm{dker}$, they tend to be more concerned with information systems as communication and sensemaking media than as social or technical control instruments. When we speak of sense-making we think of the creation and sharing of new meanings such as typically occurs in the context of judgmental decision making or social consensus formation. The sense-making function of information systems supports human actions which have the primary orientation to achieve (sincere) agreement on opinions, values, goals, means, perceptions of facts, hopes anything of shared concern. 'Sincere' means that all sides 'negotiate' in good faith, i.e. own up to their feelings, mean what they say, do not engage in manipulative tactics etc. Habermas calls this type of action 'communicative' and I defer to Jørgensens paper for further explanation of communicative action.

In distinction to this, the control functions of information systems are concerned with the efficient and effective achievement of predefined goals which are presumed to be taken for granted. While the research emphasis on sense-making phenomena is hardly surprising when approaching the research reported here from a semiotic/linguistic perspective, it stands in startling contrast to the mainstream literature in Information Systems as we know it from the journals specializing in information systems research. Most information systems research as published to-date, tends to focus on the control functions of information systems in the context of economic and technical concerns for improving efficiency or effectiveness. Only very recently has this been changing, in particular in the research specialization of group support systems or CSCW (cooperative support of cooperative work) as it is sometimes called. 
To illustrate the proposed distinction of information systems roles as sensemaking media and control instruments further, consider the difference between instrumental and strategic actions in organizations. Both of these presume that the actors are primarily success-oriented to achieve goals which are taken for granted. In strategic action we are conscious of the fact that we deal with one or more rational opponents who in turn try to control us, similar to a game in which only one party can win. In this type of situation counter-measures have to be anticipated and the consequences of one's own action evaluated in light of possible counter-measures. In instrumental action, people are looked upon as willing means which follow orders. An example might be smoothly functioning 'operating units' (as Boguslaw 1965 discusses) like a bureaucratic office department working under a supervisor or an assembly line. Of course, in real life ambiguous situations may occur. There is one paper which addresses system control (Bærentsen), but as will become clear it does not address the instrumental or strategic aspects of control. Examples of sense-making support are all systems devised to dissolve ambiguity, i.e. many decision or executive support systems, planning information systems, marketing research information systems and the like. $T$ expand on this it is helpful to distinguish the following three sense-making situations and relate them the contents of this book:

- Making sense of facts and opinions, complex situations, trends and the like. A good example is Schmidt's paper on diagnostic work or Bærentsen's analysis of the cognitive processes which allow experts to sort out confusing situations in control of complex systems.

- Interpreting shared norms or laws as typically happens in a court of law. Examples might be a loan processing system which helps a financial officer to determine if a loan application meets company guidelines and policies for granting loans or a legal information retrieval system which help to match a new case to applicable precedence and legal principles.

- Making sense of complex sequences of arguments and counter- arguments when a group of people such as a jury is in search for answers to questions of truth, justice (fairness) or good taste and no clear norms or principles apply or when the grounds for such norms themselves are at stake. Examples are some decision room applications, 'virtual committee' or conferencing systems or the research on dialectical information systems .

There are at least two papers which are directly concerned with taxonomical issues of this type. Both Klein and Truex as well as Jørgensen bear on the issue how the range of sense-making activities could be conceptualized. In part they use the same social theories, i.e. Habermas' theory of Communicative Action. 
Stamper's paper outlines Gibson's theory of sense data interpretation. The last part of the paper can be interpreted as an attempt to capture the shared norms of reality interpretation in ontological models which map the structure of information systems. Clarke provides concrete evidence how the intellectual structure of obsolete information systems 'stays behind' in the minds of users and continues to affect their sense-making.

It is clear from these examples that the contribution or impact of information systems on organizational sense-making occupies the foreground of the research presented in this book. This impression is confirmed if one considers how the papers can be grouped around common ideas, research questions or observations. In this way, selected papers can form natural pairs or triples complementing each other in contributing to common substantive questions by shedding light on related empirical phenomena. For example, Joergensen speaks in the latter half of his paper about the way in which communication constitutes the nerve and pulsating heartbeat of the organization. He points to greetings, coordinations of tasks, reminders and the like. Both Klein, Truex and Bærentsen add more finely grained empirical detail how this manifests itself. Of course, both papers invite the reader to reflect the reported examples of language use to the notions of self-reference and self-organization. Andersen, in his introductory piece singles out the stabilizing force of signs, Stamper suggests the details of a symbolism by which stable regularities can be used to capture organizational conventions in 'ontological models'. Thereby information systems can become stabilizers by freezing certain sign constellations. A similar inference follows from Klein and Truex claim that structures can be 'frozen' (kept artificially stable) by the fiat of power (but unlike Stamper they do not suggest the details of a modeling mechanism by which this might be accomplished). Hørlück gives examples for ways in which computer systems (EDI in his case) 'freeze' certain communication patters and how this robs the organization of flexibility. Bærentsen observes a similar mechanism in the design of DSS where canned solutions become incomprehensible to humans and thereby may obstruct rather than facilitate shared sense-making. Klein and Truex point to the importance of interpretations in emergence, but leave out where the ideas originate which become the subject of shared sense-making. Bærentsen's concept of episodic knowledge and memory could contribute some details of the dynamics of emergence: it points to the episodic memory as a possible link to the environment from which unpredictable events intrude into the linguistic processes described by Truex and Klein.

In summary then all papers are analytical, because they attempt to develop a theoretical grounding for a scientific problem which is most often related to self-organization. But since self-organization is also a value they tend to have conceptual critical implications and practical political consequences. These are not always made as explicit as they could be and hopefully the preceding 
examples will help to see these aspects more clearly. Furthermore, I have tried to explain why the approaches taken by the researchers contributing to this volume could help to overcome the fundamental paradigmatic deadlock which currently exists in the Information Research Community. As long as the advocates of fundamentally opposed methodological positions (e.g. positivism vs. constructivism) recognize that each has its peculiar weaknesses, it should be easy to reinterpret the situation as an opportunity for mutual learning on matters of research strategy and methodology. The following papers provide a wide spectrum of new view points which can serve as bridge heads for venturing forth into uncharted territories.

\section{References}

APPLEGATE, LYNDA M.(1994). Managing in an information age: transforming the organization for the 1990's. In: Transforming Organizations with Information Technology, ed. By R. Baskerville et al., IFIP Transactions A-49, North Holland, 1994: 15-94.

BASKERVILLE, R., J. TRAVIS, D. TRUEX (1992). Systems without method: The impact of new technologies on information systems development projects. In: Impact of Computer Supported Technologies on Information Systems Development, ed. By K. E. Kendall, K. Lyytinen and J. I. DeGross, IFIP Transactions A-8, North Holland, 1992: 241 - 269.

BOGUSLAW, R.(1965).The New Utopians - A Study of Systems Design and Social Change. Prentice Hall, Englewood Cliffs.

HIRSCHHEIM, KLEIN, LYYTINEN (1995). Information Systems Development, Conceptual and Philosophical Foundations. Cambridge Univ. Press.

NISSEN, H.-E., H.K. KLEIN AND R.A. HIRSCHHEIM, eds. (1991). Information Systems Research: Contemporary Approaches \& Emergent Traditions. Amsterdam: North-Holland.

HIRSCHHEIM, R. \& H. K. KLEIN (1994). Emancipation through ISD: The Case for ETHICS., MISQ, March 1994 (vol 18, no 1.): 83-109.

LYYTINEN, K. (1992). Information systems and critical theory. In: Critical Management Studies. Alvesson, M., \& Willmott, H. (Eds.), Sage Publications: London, 1992.

TRUEX, D. \& H. K. KLEIN (1991). A rejection of structure as a basis for information systems development. In: Collaborative Work, Social Communications and Information Systems, ed. By R. K. Stamper et al., Proc. Of the IFIP TC8 Working Conf. COSCIS 91, Helsinki, North Holland, 27-29 Aug. 1991: 213-235.

TRUEX, D. (1993). Information Systems Development in the Emergent Organization. Ph.D. Dissertation, Thomas J. Watson, School of Engineering, SUNY, Binghamton, New York.

WINOGRAD, T. \& FLORES, F. (1987). Understanding Computers and Cognition. Norwood, Reading, Mass., Addison Wesley. 
